\title{
Depth-resolved microspectroscopy of porous silicon multilayers
}

\author{
S. Manotas and F. Agulló-Ruedaa) \\ Instituto de Ciencia de Materiales de Madrid (CSIC), Cantoblanco, E-28049 Madrid, Spain \\ J. D. Moreno, R. J. Martín-Palma, R. Guerrero-Lemus, and J. M. Martínez-Duart \\ Departamento de Física Aplicada, Universidad Autónoma de Madrid, Cantoblanco, E-28049 Madrid, Spain
}

(Received 2 April 1999; accepted for publication 22 June 1999)

\begin{abstract}
We have measured depth-resolved microphotoluminescence (PL) and micro-Raman spectra on the cross section of porous silicon multilayers to sample different layer depths. The PL emission band gets stronger, blueshifts, and narrows at the high porosity layers. On the contrary, the Raman band weakens and broadens. This band is fitted to the phonon confinement model. With the bulk silicon phonon frequency and its linewidth as free parameters, we obtain crystallite size, temperature, and stress as a function of depth. Sizes are larger than those estimated from PL. Laser power was reduced to eliminate heating effects. Compressive stresses in excess of $10 \mathrm{kbar}$ are found in the deepest layer due to the lattice mismatch with the substrate. (C) 1999 American Institute of Physics. [S0003-6951(99)02833-8]
\end{abstract}

The refractive index of porous silicon can be varied continuously between the indices of bulk silicon and air, simply by changing the porosity. Therefore, porous silicon multilayers, ${ }^{1}$ where the porosity varies periodically with depth, have been used in optoelectronic devices such as filters, ${ }^{1}$ dielectric mirrors, ${ }^{2}$ waveguides, ${ }^{3}$ and optical microcavities. ${ }^{4}$ For these applications it is very important to characterize the device with depth resolution, but reports have been scarce. ${ }^{5}$ For example, Raman microspectroscopy on a cross section has been applied previously to porous silicon single layers. ${ }^{6}$ However, experiments in multilayers have been limited to top and bottom surface measurements. ${ }^{7}$

In this letter we report microphotoluminescence (PL) and micro-Raman spectra taken on the cross section of porous silicon multilayers to get direct depth-resolved information. We will find important parameters such as crystallite size, temperature, and stress as a function of depth and porosity.

Porous silicon multilayers were etched in the dark on (001) boron-doped ( $p$-type, $0.1-0.5 \Omega \mathrm{cm}$ ) silicon wafers with a 1:1 HF (48\% in weight)/ethanol (98\%) solution. Several multilayered samples with different porosities were formed by alternatively switching the current density (etching time) between two values: 20(120)/200(20), 40(100)/ $150(30)$, and $20(100) / 75(30)$ in $\mathrm{mA} / \mathrm{cm}^{2}$ (s). Estimated porosities (thicknesses in $\mu \mathrm{m}$ ) are, ${ }^{8}$ respectively, $57 \%(2.4) /$ $97 \%(2.5), 61 \%(3.4) / 86 \%(3.0)$, and $57 \%(2.0) / 69 \%(1.7)$. In the following, we will concentrate on the second sample. Samples were cleaved along a (110) plane to expose a cross section perpendicular to the layers (see Fig. 1).

PL and Raman spectra were taken along the cross section with a Renishaw ramascope 2000 microspectrometer. To sample different depths, a motorized stage was used. The exciting wavelength was $514.5 \mathrm{~nm}$ from an argon ion laser. To avoid laser heating, power density on the sample was smaller than $2 \mathrm{~kW} / \mathrm{cm}^{2}$. Spatial resolution was around $1 \mu \mathrm{m}$.

Figure 2 shows the micro-PL and micro-Raman spectra

a)Electronic mail: far@icmm.csic.es taken at different layer depths in a three-dimensional view. Porous layers extend down $28 \mu \mathrm{m}$. In contrast to the substrate they emit some luminescence and the Raman peak is broadened.

Figure 3 gathers the most relevant parameters from the PL [Figs. 3(a)-3(c)] and the Raman [Figs. 3(d) $-3(\mathrm{~g})$ ] spectra. In this sample the integrated PL intensity [Fig. 3(a)] for the low porosity (LP) layers is much weaker than for the high porosity (HP) layers, because their porosity is very close to the threshold of $65 \%$ for light emission. ${ }^{9}$

The PL emission band peaks at $1.82 \mathrm{eV}(1.78 \mathrm{eV})$ for the HP (LP) layers [Fig. 3(b)]. The shift with porosity agrees with the origin of PL at quantum states confined in the silicon nanocrystals. Assuming nanospheres, ${ }^{10}$ the energies correspond to diameters of 3.28 and $3.35 \mathrm{~nm}$ for the HP and LP layers, respectively. Within the layers the energy varies monotonically, indicating a positive (negative) gradient of the porosity with depth for the HP (LP) layers.

The PL full width at half maximum (FWHM) [Fig. 3(c)] reaches a maximum of around $0.35 \mathrm{eV}$ for LP layers and a minimum of around $0.29 \mathrm{eV}$ for HP layers. Similar narrowings of the PL band with porosity have been reported previously. ${ }^{11}$

For all samples the integrated Raman intensity [Fig. 3(d)] is stronger in the LP layers, probably due to the larger

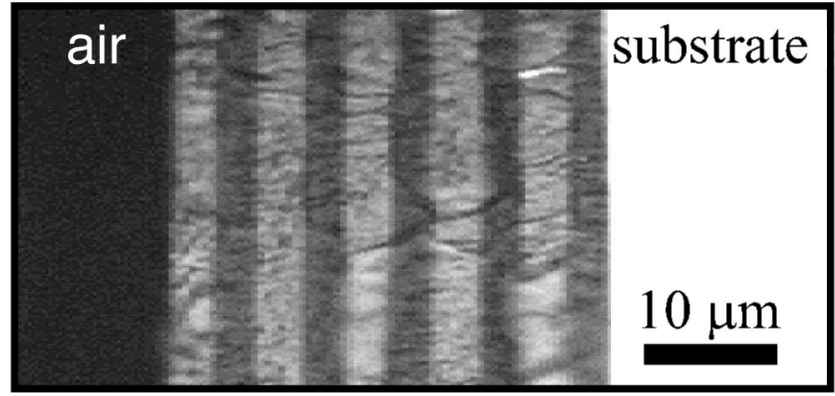

FIG. 1. Optical micrograph of a cleaved cross section for a $61 \% / 86 \%$ porous silicon multilayer. Dark bands correspond to high porosity layers. Contrast has been computer enhanced. 


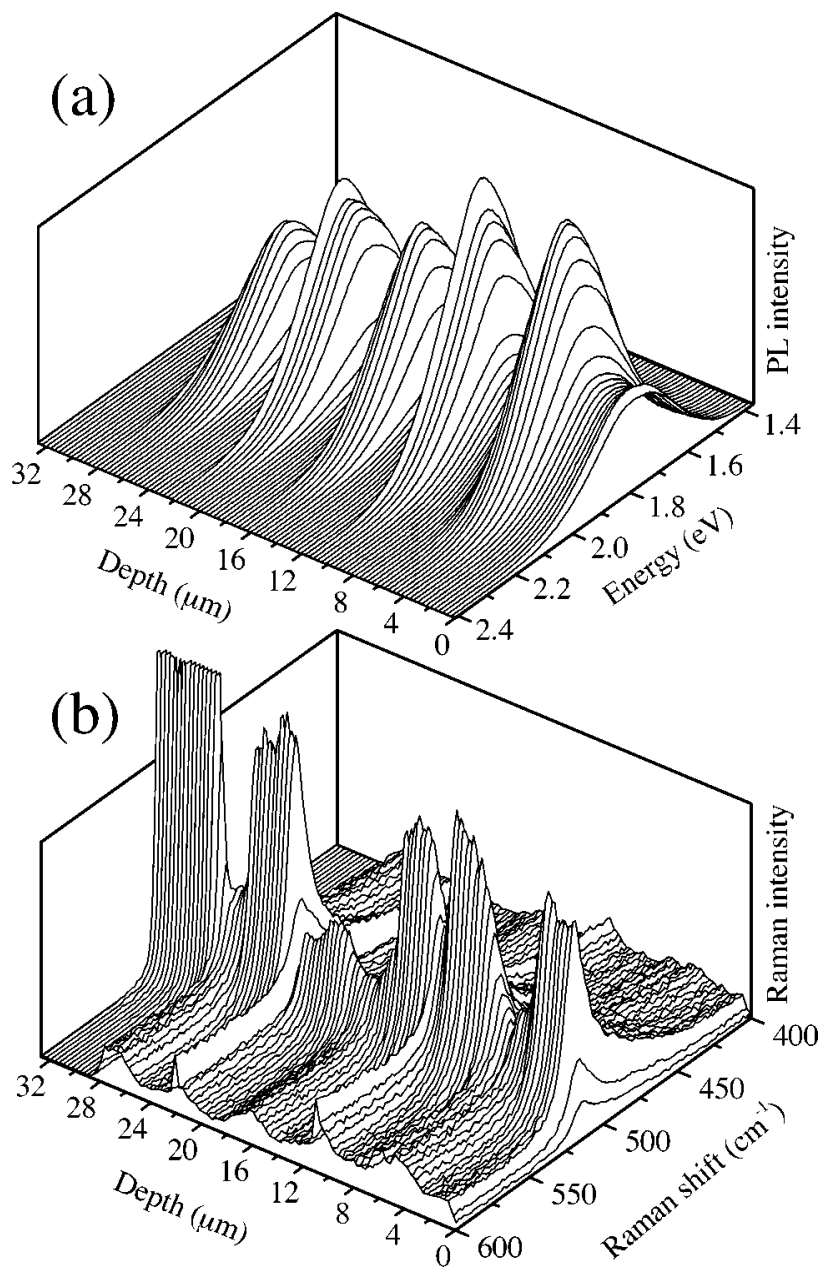

FIG. 2. 3D view of microphotoluminescence (PL) (a) and micro-Raman (b) spectra at different layer depths along the cross section shown in Fig. 1.

scattering volume. In this sample the seventh layer shows a weaker contribution due to focusing effects during the mapping, which do not affect the following discussion.

The phonon Raman band in porous silicon is broadened on the low energy side due to quantum confinement of phonons in the silicon nanocrystals and to the presence of amorphous silicon. ${ }^{12}$ Confinement relaxes the $q=0$ selection rule and phonons outside the center of the Brillouin zone $(q \neq 0)$, with lower frequency, become allowed. The resulting line shape can be related to crystallite size with the phonon confinement model. ${ }^{13,14}$

Using this model, with the usual expressions and assuming spherical nanocrystals, ${ }^{14}$ we fitted the Raman band to get the crystallite diameter as a function of depth. To account for the amorphous contribution we added a Gaussian. Its position and FWHM were left as free parameters, although we got almost constant values of 487 and $60 \mathrm{~cm}^{-1}$, respectively, corroborating the amorphous origin. The relative integrated intensity, which is proportional to the amorphous fraction, does not change with depth. To get a good agreement, the phonon parameters at $q=0$ were also varied with depth, providing new physical insight.

The phonon frequency at $q=0\left(\nu_{0}\right)$ corresponds to the bulk value. At $300 \mathrm{~K}$ it is $520 \mathrm{~cm}^{-1}$. At higher temperatures ${ }^{15}$ or under compressive stress ${ }^{16}$ it decreases or increases, respectively. For LP layers [Fig. 3(e)] the shift is increases, respectively. For LP layers [Fig. 3(e)] the shift is much more efficient.
Downloaded 10 Feb 2010 to 161.111 .180 .191 . Redistribution subject to AIP license or copyright
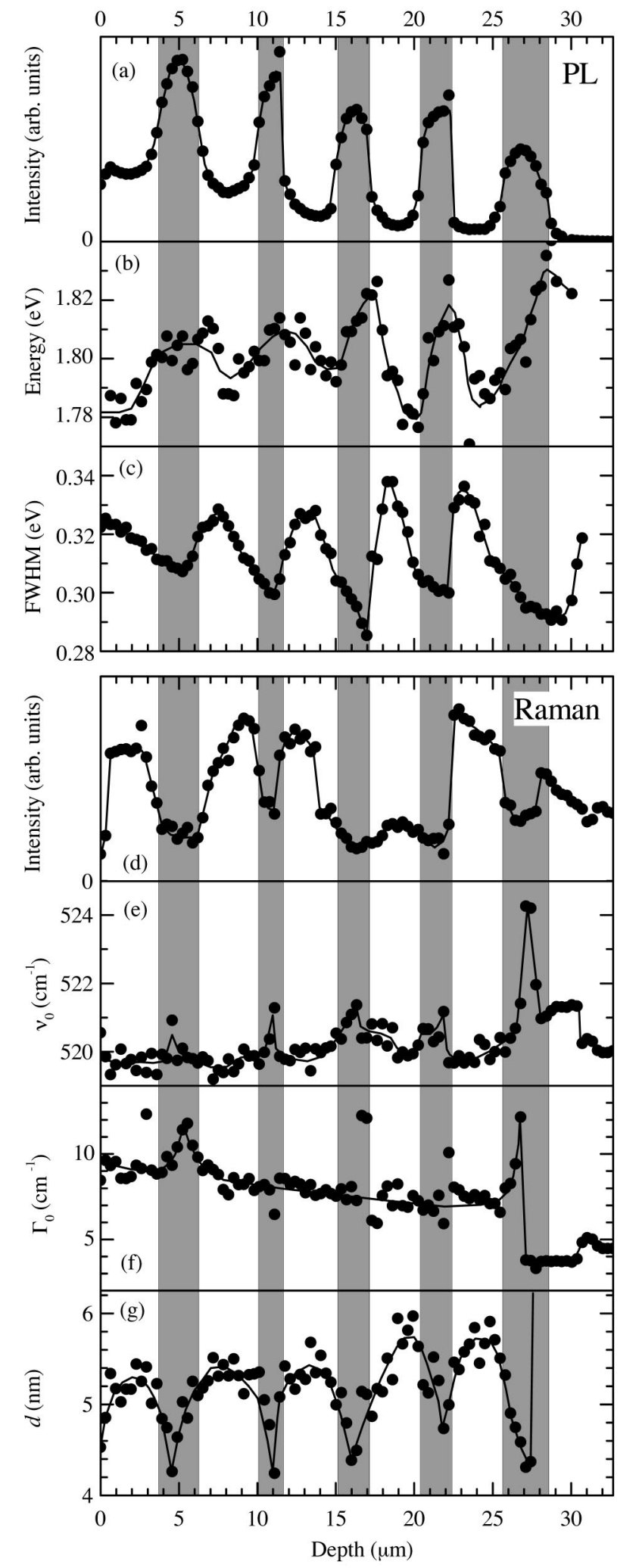

FIG. 3. Depth dependence of the most relevant parameters from the PL (a)-(c) and the Raman (d)-(g) spectra. Gray vertical stripes mark high porosity layers.

very small, proving that heating is negligible at this laser power. At higher powers $\nu_{0}$ decreases appreciably. For example, at $200 \mathrm{~kW} / \mathrm{cm}^{2}$ the shift is $4 \mathrm{~cm}^{-1}$, indicating ${ }^{15}$ a temperature of $200^{\circ} \mathrm{C}$. At this power, heating effects are still unobservable on the substrate because heat dissipation is

much more efficient.
to AIP license or copyright; see http://apl.aip.org/apl/copyright.jsp 
The lattice of porous silicon expands with porosity. ${ }^{17}$ The lattice mismatch with the substrate ${ }^{17}$ or between layers of different porosities produces a residual stress. From the observed shift of $\nu_{0}$ [Fig. 3(e)] we obtain ${ }^{16}$ compressive biaxial stresses of about 4 kbar on the HP layers and of $13 \mathrm{kbar}$ on the deepest layer, due to the lattice mismatch with the LP layers and the substrate, respectively. Considering the layer porosities, ${ }^{18}$ the substrate resistivity, ${ }^{19}$ and the elastic constants of bulk silicon, we estimate the stresses as 7 and 11 kbar, respectively, in close agreement with the experiment. The strain due to the substrate relaxes within one layer.

We observe that the phonon linewidth at $q=0\left(\Gamma_{0}\right)$ increases with temperature. ${ }^{15}$ At low laser powers, when heating effects are negligible, $\Gamma_{0}$ reaches a value in the porous layers that is higher than in the substrate [Fig. 3(f)]. We attribute the minimum $\Gamma_{0}$ to inhomogeneous broadening in the silicon microstructures. It depends on the sample, but no clear correlation is found with porosity within the same sample, in contrast with previous results. ${ }^{20}$ Porosity multilayers have the advantage that different porosities are obtained under the same conditions, other than etching time and current.

Finally, the diameter of silicon nanocrystals, $d$, obtained from the fitting, varies about $4.3-5.5 \mathrm{~nm}$ for the HP and the LP layers, respectively [Fig. 3(g)]. They are larger than those estimated from PL, because the size distribution is actually a broad function and PL is more sensitive to smaller crystals.

In summary, we have shown that low power microphotoluminescence and micro-Raman spectra on a cross section of porous silicon multilayers provide detailed information. Parameters like crystallite size, temperature, and stress can be studied as a function of depth and porosity. A large stress is found at the deepest layer.
The authors acknowledge financial support from the Spanish CICyT (Project Nos. MAT96-0395-CP and MAT970725).

${ }^{1}$ S. Frohnhoff and M. G. Berger, Adv. Mater. 6, 963 (1994).

${ }^{2}$ M. G. Berger, M. Tonissen, R. Arens-Fischer, H. Münder, H. Luth, M. Arntzen, and W. Theiss, Thin Solid Films 255, 313 (1995).

${ }^{3}$ A. Loni, L. T. Canham, M. Berger, R. Arens-Fischer, H. Munder, H. Lüth, H. F. Arrand, and T. M. Benson, Thin Solid Films 276, 143 (1996).

${ }^{4}$ V. Pellegrini, A. Tredicucci, C. Mazzoleni, and L. Pavesi, Phys. Rev. B 52, 14328 (1995).

${ }^{5}$ T. Maehama, C. Afuso, and N. Itoh, Jpn. J. Appl. Phys., Part 1 37, 998 (1998).

${ }^{6}$ K. Kozlowski and W. Lang, J. Appl. Phys. 72, 5401 (1992).

${ }^{7}$ S. Billat, M. Thonissen, R. Arensfischer, M. G. Berger, M. Kruger, and H. Luth, Thin Solid Films 297, 22 (1997).

${ }^{8}$ See, e. g., M. G. Berger, S. Frohnhoff, W. Theiss, U. Rossow, and H. Münder, in Porous Silicon Science and Technology, edited by J.-C. Vial and J. Derrien (Les Editions de Physique, Springer, 1995).

${ }^{9}$ S. Hilbrich, W. Theiß, R. Arens-Fischer, O. Glück, and M. G. Berger, Thin Solid Films 276, 231 (1996).

${ }^{10}$ C. Delerue, G. Allan, and M. Lannoo, Phys. Rev. B 48, 11024 (1993).

${ }^{11}$ P. D. J. Calcott, in Properties of Porous Silicon, edited by L. T. Canham (The Institution of Electrical Engineers, London, 1997), p. 203.

${ }^{12}$ S. R. Goodes, T. E. Jenkins, M. I. J. Beale, J. D. Benjamin, and C. Pickering, Semicond. Sci. Technol. 3, 483 (1988); R. Tsu, H. Shen, and M. Dutta, Appl. Phys. Lett. 60, 112 (1992); Z. Sui, P. P. Leong, I. P. Herman, G. S. Higashi, and H. Temkin, ibid. 60, 2086 (1992); J. C. Tsang, M. A. Tischler, and R. T. Collins, ibid. 60, 2279 (1992).

${ }^{13}$ R. J. Nemanich, S. A. Solin, and R. M. Martin, Phys. Rev. B 23, 6348 (1981); H. Richter, Z. P. Wang, and L. Ley, Solid State Commun. 39, 625 (1981).

${ }^{14}$ I. H. Campbell and P. M. Fauchet, Solid State Commun. 58, 739 (1986).

${ }^{15}$ T. R. Hart, R. L. Aggarwal, and B. Lax, Phys. Rev. B 1, 638 (1970).

${ }^{16}$ E. Anastassakis, A. Pinczuk, E. Burstein, F. H. Pollak, and M. Cardona, Solid State Commun. 8, 133 (1970).

${ }^{17}$ K. Barla, R. Herino, G. Bomchil, J. C. Pfister, and A. Freund, J. Cryst. Growth 68, 727 (1984).

${ }^{18}$ D. Bellet and G. Dolino, Thin Solid Films 276, 1 (1996).

${ }^{19}$ H. Sugiyama and O. Nittono, J. Cryst. Growth 103, 156 (1990).

${ }^{20}$ H. Münder, C. Andrzejak, M. G. Berger, U. Klemradt, H. Lüth, R. Herino, and M. Ligeon, Thin Solid Films 221, 27 (1992). 